\title{
Radiative rates and electron impact excitation rates for H-like Ar xvIII
}

\author{
K. M. Aggarwal ${ }^{1}$, K. Hamada ${ }^{2}$, A. Igarashi ${ }^{2}$, V. Jonauskas ${ }^{3}$, F. P. Keenan ${ }^{1}$, and S. Nakazaki ${ }^{2}$ \\ 1 Astrophysics Research Centre, School of Mathematics and Physics, Queen's University Belfast, Belfast BT7 1NN, Northern Ireland, \\ UK \\ e-mail: K.Aggarwal@qub.ac.uk \\ 2 Department of Applied Physics, Faculty of Engineering, University of Miyazaki, Miyazaki 889-2192, Japan \\ 3 Vilnius University Research Institute of Theoretical Physics and Astronomy, A. Goštauto 12, 01108 Vilnius, Lithuania
}

Received 17 April 2008 / Accepted 22 May 2008

\section{ABSTRACT}

\begin{abstract}
Aims. In this paper we report on calculations for energy levels, radiative rates, collision strengths, and effective collision strengths for transitions among the lowest 25 levels of the $n \leq 5$ configurations of H-like Ar XVIII.

Methods. The general-purpose relativistic atomic structure package (GRASP) and Dirac atomic $R$-matrix code (DARC) are adopted for the calculations.

Results. Radiative rates, oscillator strengths, and line strengths are reported for all electric dipole (E1), magnetic dipole (M1), electric quadrupole (E2), and magnetic quadrupole (M2) transitions among the 25 levels. Furthermore, collision strengths and effective collision strengths are listed for all 300 transitions among the above 25 levels over a wide energy (temperature) range up to 800 Ryd $\left(10^{7.4} \mathrm{~K}\right)$.
\end{abstract}

Key words. atomic data - atomic processes

\section{Introduction}

Recently, a wealth of high resolution spectra in the UV, EUV and X-ray regions have been obtained for solar, stellar and other astrophysical sources by many space missions, such as SOHO, Chandra and XMM-Newton. Many of the observed emission lines are due to highly ionized argon, and some of these from Ar XIV-XVIII have been listed by Dere et al. (2001). A complete list of lines over a wide range of wavelengths for many ions, including those of argon, are available in the CHIANTI database at http://wwwsolar.nrl.navy.mil/ chianti.html. Additionally, some of the lines from H-like Ar XVIII, particularly in the 2.8-900 $\AA$ wavelength range, are listed in the Atomic Line List (v2.04) of Peter van Hoof at http://www . pa.uky . edu/ peter/atomic/. Atomic data are required for the generation of reliable synthetic spectra, and a line list helps in the proper identification of observed emission or absorption features.

An analysis of observed spectra provides information on the temperature, density and chemical composition of the plasmas. However, such an analysis requires information for a wide range of atomic parameters, such as energy levels, radiative rates, and excitation rate coefficients. Therefore, with this in mind we have already reported calculations for Ar XIII-XV (Aggarwal et al. 2005), Ar XVI (McKeown et al. 2004) and Ar XVII (Aggarwal \& Keenan 2005). In this paper we report similar results for transitions in H-like Ar XVIII.

Apart from energy levels, there is a paucity of measurements for the above atomic parameters for Ar XVIII, and hence

* Tables 2-4 are only available in electronic form at the CDS via anonymous ftp to cdsarc.u-strasbg.fr $(130.79 .128 .5)$ or via http://cdsweb.u-strasbg.fr/cgi-bin/qcat? J/A+A/487/383 theoretical results are of vital importance. Furthermore, to our knowledge, no calculation exists which includes a large number of transitions. Therefore, in this work we report results for a greater number of energy levels, and hence for correspondingly larger set of transitions. Additionally, we report radiative rates ( $A$-values) for all allowed and intercombination, i.e. electric dipole (E1), electric quadrupole (E2), magnetic dipole (M1), and magnetic quadrupole (M2) transitions, as these data are required in the modelling of plasmas. For the generation of wavefunctions we adopt the fully relativistic GRASP (general-purpose relativistic atomic structure package) code of Grant et al. (1980), which has been updated by Dr. Norrington. Similarly, for computations of collision strengths $(\Omega)$ and subsequently of effective collision strengths $\Upsilon$, we adopt the Dirac atomic $R$-matrix code (DARC) of Norrington \& Grant (private communication).

\section{Energy levels}

The $n \leq 5$ configurations of Ar XVIII give rise to 25 fine-structure levels, listed in Table 1. Our calculated energies obtained from the GRASP code, with and without including the QED effects, are given in this table along with those from the experimental compilation of NIST (http://physics.nist.gov/PhysRefData). For our calculations, we have used the option of extended average level (EAL), in which a weighted (proportional to $2 j+1$ ) trace of the Hamiltonian matrix is minimized. This produces a compromise set of orbitals describing closely lying states with moderate accuracy. The inclusion of QED effects lowers the energies by less than 0.1 Ryd, but brings these slightly closer to the experimental results. In the case of Coulomb energies, levels with same $n$ and angular momentum $J$ (such as 2/3 and $5 / 6$ ) are quasi-degenerate, but split with the inclusion of QED 
Table 1. Energy levels (in Ryd) of Ar XVIII.

\begin{tabular}{rllrrrr}
\hline \hline Index & Configuration & Level & NIST & GRASP $^{a}$ & GRASP $^{b}$ & FAC $^{c}$ \\
\hline 1 & $1 \mathrm{~s}$ & ${ }^{2} \mathrm{~S}_{1 / 2}$ & 0.00000 & 0.00000 & 0.00000 & 0.0000 \\
2 & $2 \mathrm{~s}$ & ${ }^{2} \mathrm{~S}_{1 / 2}$ & 243.89332 & 243.96899 & 243.89703 & 243.9086 \\
3 & $2 \mathrm{p}$ & ${ }^{2} \mathrm{P}^{\circ}{ }^{1 / 2}$ & 243.88146 & 243.96899 & 243.88553 & 243.8974 \\
4 & $2 \mathrm{p}$ & ${ }^{2} \mathrm{P}^{\circ}{ }_{3 / 2}$ & 244.23540 & 244.32219 & 244.23949 & 244.2513 \\
5 & $3 \mathrm{p}$ & ${ }^{2} \mathrm{P}^{\circ}{ }_{1 / 2}$ & 289.16523 & 289.25314 & 289.16989 & 289.1835 \\
6 & $3 \mathrm{~s}$ & ${ }^{2} \mathrm{~S}_{1 / 2}$ & 289.16878 & 289.25314 & 289.17334 & 289.1868 \\
7 & $3 \mathrm{~d}$ & ${ }^{2} \mathrm{D}_{3 / 2}$ & 289.26995 & 289.35785 & 289.27469 & 289.2882 \\
8 & $3 \mathrm{p}$ & ${ }^{2} \mathrm{P}^{\circ}{ }_{3 / 2}$ & 289.27013 & 289.35785 & 289.27481 & 289.2884 \\
9 & $3 \mathrm{~d}$ & ${ }^{2} \mathrm{D}_{5 / 2}$ & 289.30464 & 289.39246 & 289.30930 & 289.3228 \\
10 & $4 \mathrm{p}$ & ${ }^{2} \mathrm{P}^{\circ}{ }_{1 / 2}$ & 305.00012 & 305.08820 & 305.00504 & 305.0192 \\
11 & $4 \mathrm{~s}$ & ${ }^{2} \mathrm{~S}_{1 / 2}$ & 305.00163 & 305.08820 & 305.00647 & 305.0206 \\
12 & $4 \mathrm{~d}$ & ${ }^{2} \mathrm{D}_{3 / 2}$ & 305.04428 & 305.13235 & 305.04922 & 305.0634 \\
13 & $4 \mathrm{p}$ & ${ }^{2} \mathrm{P}^{\circ}{ }_{3 / 2}$ & 305.04437 & 305.13235 & 305.04926 & 305.0634 \\
14 & $4 \mathrm{~d}$ & ${ }^{2} \mathrm{D}_{5 / 2}$ & 305.05893 & 305.14697 & 305.06381 & 305.0779 \\
15 & $4 \mathrm{f}$ & ${ }^{2} \mathrm{~F}^{\circ}{ }_{5 / 2}$ & 305.05890 & 305.14697 & 305.06381 & 305.0779 \\
16 & $4 \mathrm{f}$ & ${ }^{2} \mathrm{~F}^{\circ}{ }_{7 / 2}$ & 305.06620 & 305.15427 & 305.07111 & 305.0852 \\
17 & $5 \mathrm{p}$ & ${ }^{2} \mathrm{P}^{\circ}{ }_{1 / 2}$ & 312.32326 & 312.41144 & 312.32828 & 312.3427 \\
18 & $5 \mathrm{~s}$ & ${ }^{2} \mathrm{~S}_{1 / 2}$ & 312.32403 & 312.41144 & 312.32901 & 312.3434 \\
19 & $5 \mathrm{~d}$ & ${ }^{2} \mathrm{D}_{3 / 2}$ & 312.34587 & 312.43402 & 312.35089 & 312.3653 \\
20 & $5 \mathrm{p}$ & ${ }^{2} \mathrm{P}^{\circ}{ }_{3 / 2}$ & 312.34590 & 312.43402 & 312.35092 & 312.3653 \\
21 & $5 \mathrm{f}$ & ${ }^{2} \mathrm{~F}^{\circ}{ }_{5 / 2}$ & 312.35335 & 312.44150 & 312.35837 & 312.3728 \\
22 & $5 \mathrm{~d}$ & ${ }^{2} \mathrm{D}_{5 / 2}$ & 312.35336 & 312.44150 & 312.35837 & 312.3728 \\
23 & $5 \mathrm{~g}$ & ${ }^{2} \mathrm{G}_{7 / 2}$ & 312.35708 & 312.44525 & 312.36209 & 312.3765 \\
24 & $5 \mathrm{f}$ & ${ }^{2} \mathrm{~F}^{\circ}{ }_{7 / 2}$ & 312.35708 & 312.44525 & 312.36209 & 312.3765 \\
25 & $5 \mathrm{~g}$ & ${ }^{2} \mathrm{G}_{9 / 2}$ & 312.35993 & 312.44748 & 312.36432 & 312.3788 \\
\hline
\end{tabular}

NIST: http://physics.nist.gov/PhysRefData.

${ }^{a}$ Coulomb energies; ${ }^{b}$ QED corrected energies; ${ }^{c}$ energies calculated from the FAC code.

effects (Lamb shift). As a result, the level orderings change slightly. However, we have retained the original orderings of the Coulomb energies, as these are the ones adopted in the subsequent tables. In general, the theoretical energies agree very well with the experimental values, both in magnitude and orderings.

\section{Radiative rates}

The absorption oscillator strength $\left(f_{i j}\right)$ and radiative rate $A_{j i}$ (in $\mathrm{s}^{-1}$ ) for a transition $i \rightarrow j$ are related by the following expression:

$f_{i j}=\frac{m c}{8 \pi^{2} e^{2}} \lambda_{j i}^{2} \frac{\omega_{j}}{\omega_{i}} A_{j i}=1.49 \times 10^{-16} \lambda_{j i}^{2}\left(\omega_{j} / \omega_{i}\right) A_{j i}$

where $m$ and $e$ are the electron mass and charge, respectively, $c$ is the velocity of light, $\lambda_{j i}$ is the transition energy/wavelength in $\AA$, and $\omega_{i}$ and $\omega_{j}$ are the statistical weights of the lower $i$ and upper $j$ levels, respectively. Similarly, the oscillator strength $f_{i j}$ (dimensionless) and the line strength $S$ (in atomic unit, 1 au = $6.460 \times 10^{-36} \mathrm{~cm}^{2} \mathrm{esu}^{2}$ ) are related by the following standard equations.

For the electric dipole (E1) transitions

$A_{j i}=\frac{2.0261 \times 10^{18}}{\omega_{j} \lambda_{j i}^{3}} S^{\mathrm{E} 1} \quad$ and $\quad f_{i j}=\frac{303.75}{\lambda_{j i} \omega_{i}} S^{\mathrm{E} 1}$,

for the magnetic dipole (M1) transitions

$A_{j i}=\frac{2.6974 \times 10^{13}}{\omega_{j} \lambda_{j i}^{3}} S^{\mathrm{M} 1} \quad$ and $\quad f_{i j}=\frac{4.044 \times 10^{-3}}{\lambda_{j i} \omega_{i}} S^{\mathrm{M} 1}$, for the electric quadrupole (E2) transitions

$A_{j i}=\frac{1.1199 \times 10^{18}}{\omega_{j} \lambda_{j i}^{5}} S^{\mathrm{E} 2} \quad$ and $\quad f_{i j}=\frac{167.89}{\lambda_{j i}^{3} \omega_{i}} S^{\mathrm{E} 2}$,

and for the magnetic quadrupole (M2) transitions

$A_{j i}=\frac{1.4910 \times 10^{13}}{\omega_{j} \lambda_{j i}^{5}} S^{\mathrm{M} 2} \quad$ and $\quad f_{i j}=\frac{2.236 \times 10^{-3}}{\lambda_{j i}^{3} \omega_{i}} S^{\mathrm{M} 2}$.

In Table 2 we present transition energies $\left(\Delta E_{i j}\right.$ in $\AA$ ), radiative rates $\left(A_{j i}\right.$ in s $\left.{ }^{-1}\right)$, oscillator strengths ( $f_{i j}$, dimensionless), and line strengths $(S$ in au), in length form only, for all 90 electric dipole (E1) transitions among the 25 levels of Ar XVIII. The indices used to represent the lower and upper levels of a transition have already been defined in Table 1 . However, for the 107 electric quadrupole (E2), 86 magnetic dipole (M1) and 108 magnetic quadrupole (M2) transitions only the $A$-values are listed. Corresponding results for the $f$ - and $S$-values can be obtained by using the above equations.

The only other results available in the literature with which to compare are those listed on the CHIANTI database for (most of) the E1 transitions. These $A$-values have been determined from the calculations of Parpia \& Johnson (1982), and there are no discrepancies with the present results for any of the transitions in common. Furthermore, we have performed another calculation from the Flexible Atomic Code (FAC) of Gu (2003), which is available from the website http://kipac-tree.stanford. $\mathrm{edu} / \mathrm{fac}$. This is also a fully relativistic code which provides a variety of atomic parameters, and yields results comparable to GRASP and DARC, particularly for highly ionized elements and at higher energies. Thus results from FAC are helpful in assessing the accuracy of atomic parameters, and for the transitions 


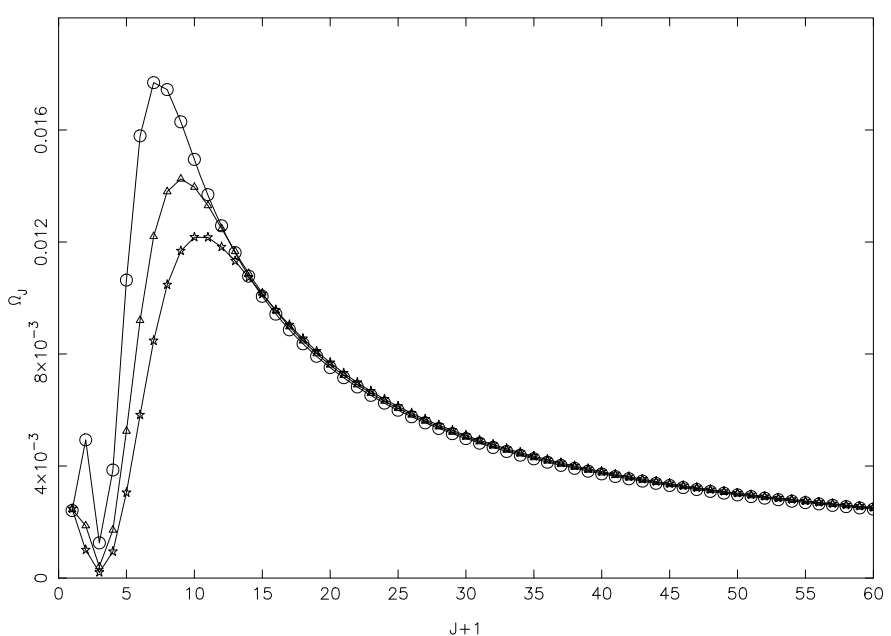

Fig. 1. Partial collision strengths for the $2 s^{2} S_{1 / 2}-2 p{ }^{2} P_{1 / 2}^{\circ}(2-3)$ transition of Ar XVIII, at three energies of: 400 Ryd (circles), 600 Ryd (triangles), and 800 Ryd (stars).

listed in Table 2 there is no discrepancy between the two sets of $A$-values from the FAC and GRASP codes. Therefore, we may confidently state that the $A$-values listed in Table 2 are accurate to better than $5 \%$.

\section{Collision strengths}

For the calculations of collision strengths, we have employed the DARC program, which includes the relativistic effects in a systematic way, in both the target description and the scattering model. It is based on the $j j$ coupling scheme, and uses the Dirac-Coulomb Hamiltonian in the $R$-matrix approach. However, because of the inclusion of fine-structure in the definition of channel coupling, the matrix size of the Hamiltonian increases substantially. The $R$-matrix radius has been adopted to be $4.80 \mathrm{au}$, and 56 continuum orbitals have been included for each channel angular momentum for the expansion of the wavefunction. This allows us to compute $\Omega$ up to an energy of 800 Ryd. The maximum number of channels for a partial wave is 110 , and the corresponding size of the Hamiltonian matrix is 6198. In order to obtain convergence of $\Omega$ for all transitions and at all energies, we have included all partial waves with angular momentum $J \leq 60$, although a higher range would have been preferable for the convergence of allowed transitions, in particular those with $\Delta n=0$. However, to account for higher neglected partial waves, we have included a top-up, based on the Coulomb-Bethe approximation for allowed transitions and geometric series for forbidden transitions.

In Figs. $1-3$ we show the variation of $\Omega$ with angular momentum $J$ at three energies of 400, 600 and 800 Ryd, and for three transitions, namely $2-3\left(2 \mathrm{~s}{ }^{2} \mathrm{~S}_{1 / 2}-2 \mathrm{p}{ }^{2} \mathrm{P}_{1 / 2}^{\circ}\right), 2-5$ $\left(2 \mathrm{~s}{ }^{2} \mathrm{~S}_{1 / 2}-3 \mathrm{p}{ }^{2} \mathrm{P}_{1 / 2}^{\circ}\right)$ and $6-7\left(3 \mathrm{~s}{ }^{2} \mathrm{~S}_{1 / 2}-3 \mathrm{~d}{ }^{2} \mathrm{D}_{3 / 2}\right)$, which are "elastic" (i.e. allowed with $\Delta n=0)$, allowed $(\Delta n \neq 0)$, and forbidden, respectively. For the forbidden and allowed transitions shown in Figs. 2 and 3, the values of $\Omega$ have fully converged at all energies, including the highest energy of our calculations. However, for the "elastic" transitions our range of partial waves is not sufficient for the convergence of $\Omega$, as shown in Fig. 1. For such transitions the top-up from the Coulomb-Bethe approximation is quite significant.

In Table 3 we present our results of $\Omega$ for all transitions over a wider energy range ( $350 \leq E \leq 800 \mathrm{Ryd})$, but above thresholds.

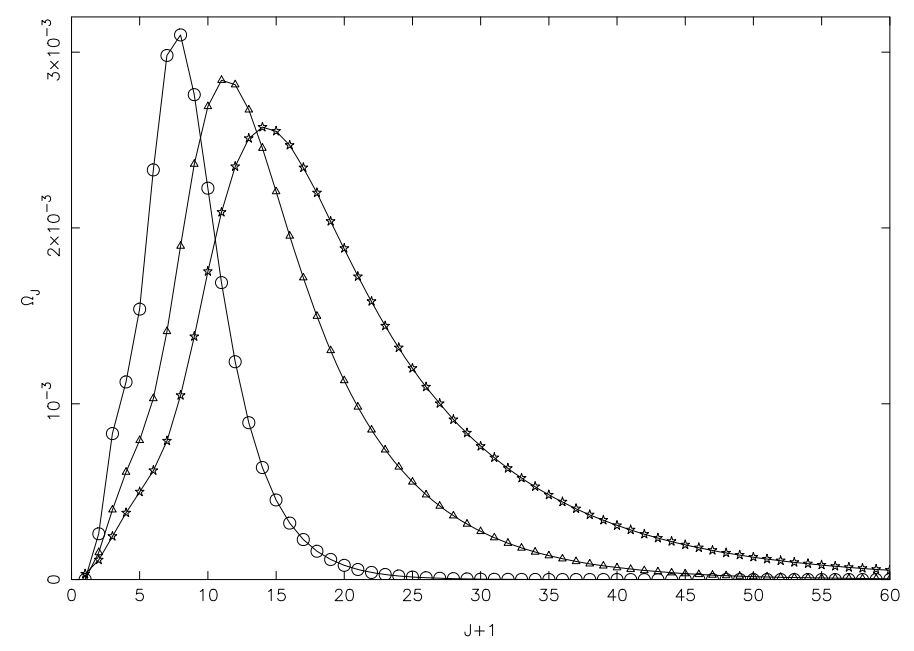

Fig. 2. Partial collision strengths for the $2 \mathrm{~s}^{2} \mathrm{~S}_{1 / 2}-3 \mathrm{p}{ }^{2} \mathrm{P}_{1 / 2}^{\circ}(2-5)$ transition of Ar XVIII, at three energies of: 400 Ryd (circles), 600 Ryd (triangles), and 800 Ryd (stars).

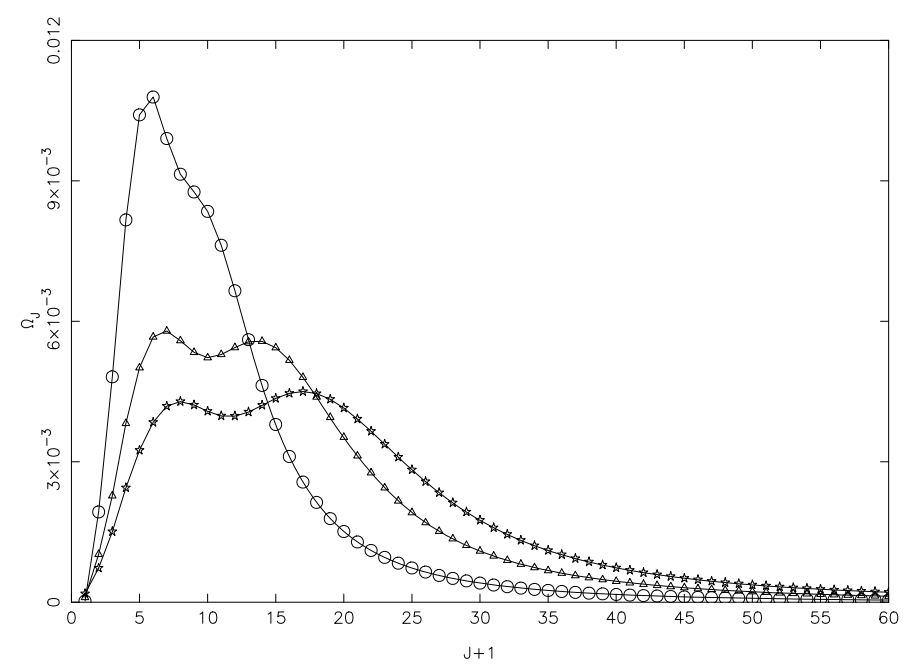

Fig. 3. Partial collision strengths for the $3 \mathrm{~s}{ }^{2} \mathrm{~S}_{1 / 2}-3 \mathrm{~d}{ }^{2} \mathrm{D}_{3 / 2}(6-7)$ transition of Ar XVIII, at three energies of: (a) 400 Ryd (circles), 600 Ryd (triangles), and 800 Ryd (stars).

The indices adopted to represent a transition are given in Table 1. These results for $\Omega$ are not directly applicable in any modelling work, but are very useful in assessing the accuracy of a calculation. Unfortunately there are no other available results with which to compare as stated already in Sect. 1. Therefore, we have performed another calculation from the FAC code.

In Table 1 we have also included the energy levels obtained from the FAC code, which are comparable with our calculations from GRASP as well as the experimental compilation. Similarly, the $A$-values obtained from FAC are also comparable with our calculations from GRASP for a majority of the E1 transitions, as already stated in Sect. 3. The values of $\Omega$ calculated from the FAC code are also listed in Table 3 at a single excited $\left(E_{j}\right)$ energy of $\sim 450 \mathrm{Ryd}$, which nearly corresponds to the highest (initial) energy of our calculations, i.e. 800 Ryd. These $\Omega$ values from FAC provide a ready comparison with our corresponding results from DARC. The two sets of $\Omega$ agree very well (generally within 10\%) for (almost) all transitions, including the weaker ones, such as $1-15\left(1 \mathrm{~s}{ }^{2} \mathrm{~S}_{1 / 2}-4 \mathrm{f}^{2} \mathrm{~F}_{5 / 2}^{\circ}\right), 1-23$ $\left(1 \mathrm{~s}^{2} \mathrm{~S}_{1 / 2}-5 \mathrm{~g}^{2} \mathrm{G}_{7 / 2}\right)$ and $1-25\left(1 \mathrm{~s}^{2} \mathrm{~S}_{1 / 2}-5 \mathrm{~g}^{2} \mathrm{G}_{9 / 2}\right)$. Similarly, for 


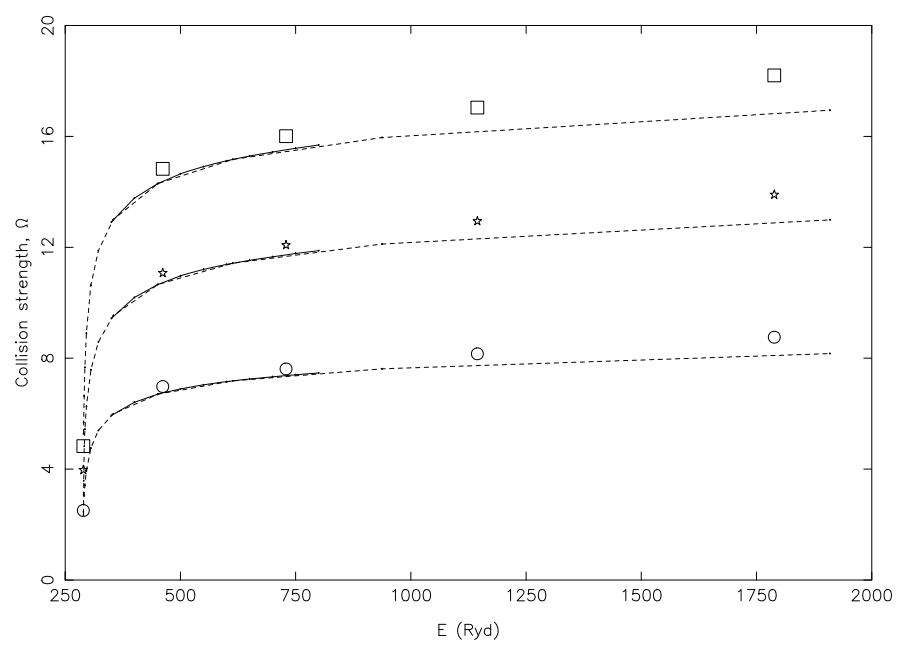

Fig. 4. Comparison of collision strengths for the 5-7 $\left(\begin{array}{llllll}3 \mathrm{p} & { }^{2} \mathrm{P}_{1 / 2}^{\circ}-3 \mathrm{~d} & { }^{2} \mathrm{D}_{3 / 2}\end{array}\right), \quad 6-8 \quad\left(3 \mathrm{~s} \quad{ }^{2} \mathrm{~S}_{1 / 2}-3 \mathrm{p} \quad{ }^{2} \mathrm{P}_{3 / 2}^{\circ}\right)$ and $8-9$ $\left(3 \mathrm{p}^{2} \mathrm{P}_{3 / 2}^{\circ}-3 \mathrm{~d}^{2} \mathrm{D}_{5 / 2}\right)$ transitions of $\mathrm{Ar}$ XVIII. Continuous curves are from our calculations from DARC, broken curves are from the $\mathrm{CC}+\mathrm{CB}$ programs, and the $\Omega$ from FAC are shown as, circles: $5-7$, stars: $6-8$ and squares: $8-9$ transition.

the allowed transitions the two sets of $\Omega$ agree within $\sim 10 \%$, as seen in Table 3 and also illustrated in Fig. 4 for three transitions, namely 5-7 (3p $\left.{ }^{2} \mathrm{P}_{1 / 2}^{\circ}-3 \mathrm{~d}^{2} \mathrm{D}_{3 / 2}\right), 6-8\left(3 \mathrm{~s}^{2} \mathrm{~S}_{1 / 2}-3 \mathrm{p}{ }^{2} \mathrm{P}_{3 / 2}^{\circ}\right)$ and $8-9\left(3 \mathrm{p}^{2} \mathrm{P}_{3 / 2}^{\circ}-3 \mathrm{~d}^{2} \mathrm{D}_{5 / 2}\right)$. However, for two transitions, namely 12-17 $\left(4 \mathrm{~d}^{2} \mathrm{D}_{3 / 2}-5 \mathrm{p}^{2} \mathrm{P}_{1 / 2}^{\circ}\right)$ and $14-20\left(4 \mathrm{~d}^{2} \mathrm{D}_{5 / 2}-5 \mathrm{p}^{2} \mathrm{P}_{3 / 2}^{\circ}\right)$, our values of $\Omega$ are higher by about a factor of two. Moreover, there are four transitions, namely $14-15\left(4 \mathrm{~d}^{2} \mathrm{D}_{5 / 2}-4 \mathrm{f}^{2} \mathrm{~F}_{5 / 2}^{\circ}\right), 19-20$ $\left(5 \mathrm{~d}^{2} \mathrm{D}_{3 / 2}-5 \mathrm{p}{ }^{2} \mathrm{P}_{3 / 2}^{\circ}\right), 21-22\left(5 \mathrm{f}^{2} \mathrm{~F}_{5 / 2}^{\circ}-5 \mathrm{~d}^{2} \mathrm{D}_{5 / 2}\right)$, and $23-24$ $\left(5 \mathrm{~g}^{2} \mathrm{G}_{7 / 2}-5 \mathrm{f}^{2} \mathrm{~F}_{7 / 2}^{\circ}\right)$, for which the differences between the FAC and DARC calculations are significant. The reason for the large differences becomes apparent when we have a closer look at the energy levels in Table 1. For all of these transitions, the energy differences $(\Delta E)$ are (almost) zero in our GRASP and FAC calculations. Transitions such as the above ones are "elastic", i.e. allowed with $\Delta n=0$ and $\Delta E \sim 0$, and converge very slowly with increasing number of partial waves, as demonstrated earlier by Igarashi et al. (2003). Since both the DARC and FAC codes include the contribution of higher neglected partial waves from the Coulomb-Bethe formulation of Burgess et al. (1970), which is highly sensitive to the adopted $\Delta E$, we obtain differing values of $\Omega$. Therefore, in order to resolve the differences between the FAC and DARC calculations, and to determine values of $\Omega$ as accurately as possible, we have performed yet another calculation using a combination of the close-coupling (CC) and CoulombBorn (CB) programs of Igarashi et al. $(2003,2005)$. These calculations are similar to those performed recently for elastic transitions in Al XIII (Aggarwal et al. 2008b) and Fe XXVI (Aggarwal et al. 2008a), and for which we have adopted the energy levels of NIST.

The results of $\Omega$ obtained from the $\mathrm{CC}+\mathrm{CB}$ program are included in Fig. 4 for the 5-7, 6-8 and 8-9 transitions, and agree very well with the other two calculations from DARC and FAC. This gives us confidence in the calculated values of $\Omega$ from the $\mathrm{CC}+\mathrm{CB}$ program. In Fig. 5 we show similar comparisons between the $\Omega$ values from the FAC and $\mathrm{CC}+\mathrm{CB}$ programs for three transitions, namely $14-15\left(4 \mathrm{~d}^{2} \mathrm{D}_{5 / 2}-4 \mathrm{f}^{2} \mathrm{~F}_{5 / 2}^{\circ}\right), 21-22$ $\left(5 \mathrm{f}^{2} \mathrm{~F}_{5 / 2}^{\circ}-5 \mathrm{~d}^{2} \mathrm{D}_{5 / 2}\right)$, and $23-24\left(5 \mathrm{~g}^{2} \mathrm{G}_{7 / 2}-5 \mathrm{f}^{2} \mathrm{~F}_{7 / 2}^{\circ}\right)$. Apart from

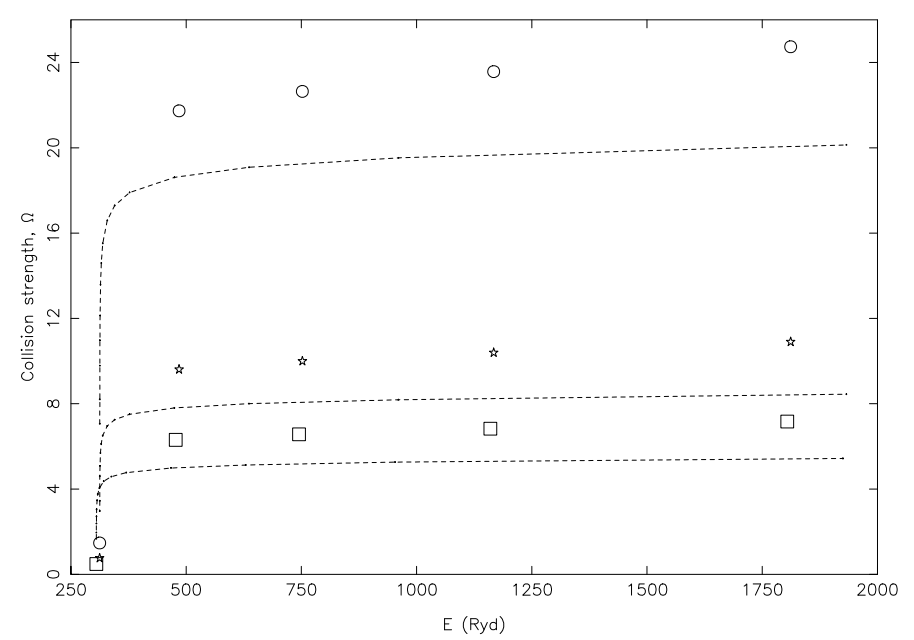

Fig. 5. Comparison of collision strengths for the 14-15 $\left(4 d^{2} \mathrm{D}_{5 / 2}-4 \mathrm{f}^{2} \mathrm{~F}_{5 / 2}^{\circ}\right), \quad 21-22$ (5f $\left.{ }^{2} \mathrm{~F}_{5 / 2}^{\circ}-5 \mathrm{~d}{ }^{2} \mathrm{D}_{5 / 2}\right)$, and $23-24$ $\left(5 \mathrm{~g}^{2} \mathrm{G}_{7 / 2}-5 \mathrm{f}^{2} \mathrm{~F}_{7 / 2}^{\circ}\right)$ transitions of $\mathrm{Ar}$ XVIII. Broken curves are from the $\mathrm{CC}+\mathrm{CB}$ programs, and the $\Omega$ from $\mathrm{FAC}$ are shown as, squares: $14-15$, circles: $21-22$ and stars: $23-24$ transition.

the lowest common energy ( $\sim 300$ Ryd), the $\Omega$ values from FAC are overestimated by up to $\sim 25 \%$, for the reasons explained above. Therefore, for the 26 elastic transitions we have adopted values of $\Omega$ from our CC+CB calculations, and from the DARC code for the remaining 274 transitions.

Since we have adopted a wide range of partial waves in order to obtain the convergence of $\Omega$ values for the forbidden as well as the allowed transitions, including the elastic ones, we estimate our results for $\Omega$ listed in Table 3 are accurate to $\sim 10 \%$. This estimate is based on a variety of comparisons made among the different calculations.

\section{Effective collision strengths}

Effective collision strengths $\Upsilon$ are obtained after integrating $\Omega$ over a Maxwellian distribution of electron velocities, i.e.

$\Upsilon\left(T_{\mathrm{e}}\right)=\int_{0}^{\infty} \Omega(E) \exp \left(-E_{j} / k T_{\mathrm{e}}\right) \mathrm{d}\left(E_{j} / k T_{\mathrm{e}}\right)$

where $E_{j}$ is the incident energy of the electron with respect to the final state of the transition, $k$ is Boltzmann's constant, and $T_{\mathrm{e}}$ is the electron temperature in $K$. Once the value of $\Upsilon$ is known for a transition, the corresponding value of the excitation $q(i, j)$ and de-excitation $q(j, i)$ rate coefficients can be easily obtained from the following simple relations:

$q(i, j)=\frac{8.63 \times 10^{-6}}{\omega_{i} T_{\mathrm{e}}^{1 / 2}} \Upsilon \exp \left(-E_{i j} / k T_{\mathrm{e}}\right) \mathrm{cm}^{3} \mathrm{~s}^{-1}$

and

$q(j, i)=\frac{8.63 \times 10^{-6}}{\omega_{j} T_{\mathrm{e}}^{1 / 2}} \Upsilon \mathrm{cm}^{3} \mathrm{~s}^{-1}$,

where $\omega_{i}$ and $\omega_{j}$ are the statistical weights of the initial $(i)$ and final $(j)$ states, respectively, and $E_{i j}$ is the transition energy.

Since the threshold energy region is dominated by numerous resonances, $\Omega$ have been computed at a large number of energies in order to delineate these resonances. We have performed 


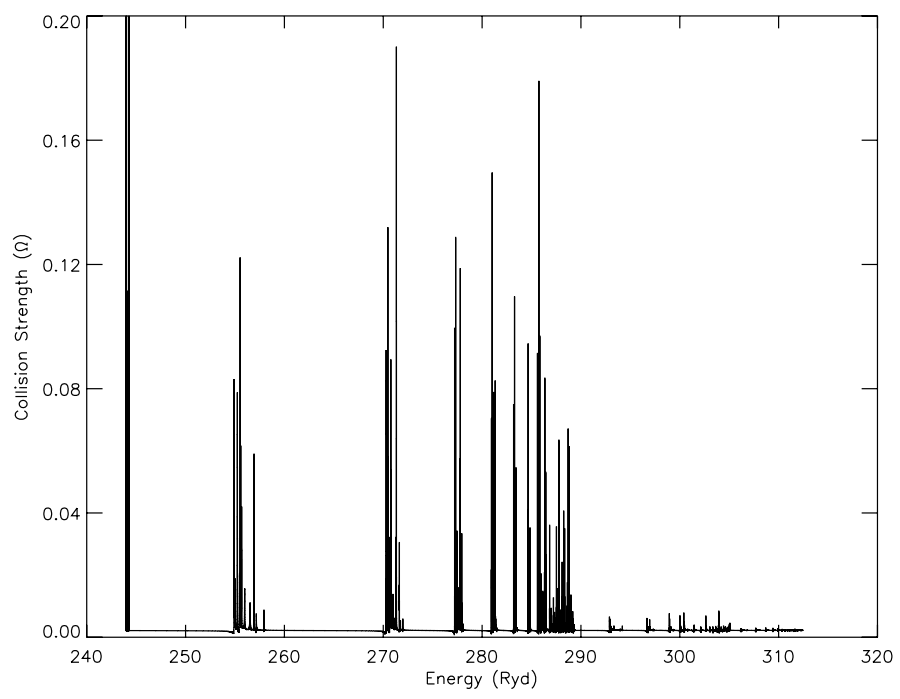

Fig. 6. Collision strengths for the $1 \mathrm{~s}^{2} \mathrm{~S}_{1 / 2}-2 \mathrm{~s}{ }^{2} \mathrm{~S}_{1 / 2}(1-2)$ transition of Ar XVIII.

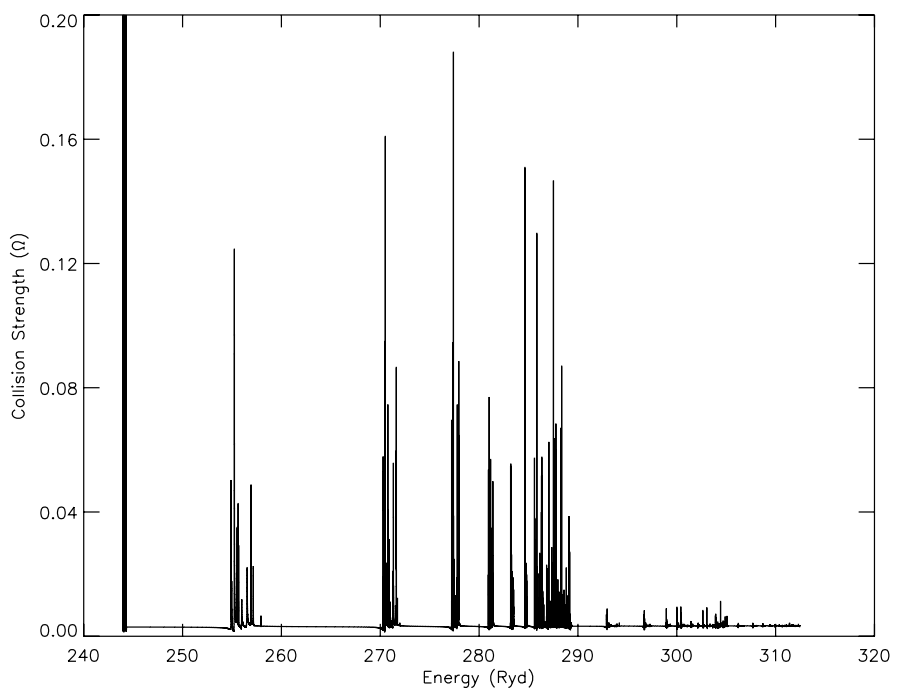

Fig. 7. Collision strengths for the $1 \mathrm{~s}^{2} \mathrm{~S}_{1 / 2}-2 \mathrm{p}{ }^{2} \mathrm{P}_{1 / 2}^{\circ}(1-3)$ transition of Ar XVIII.

our calculations of $\Omega$ at $\sim 35000$ energies in the threshold region. Close to thresholds ( $\sim 0.1$ Ryd above a threshold) the energy mesh is $0.001 \mathrm{Ryd}$, and away from thresholds is 0.002 Ryd. Thus care has been taken to include as many resonances as possible, and with as fine a resolution as is computationally feasible. The density and importance of resonances can be appreciated from Figs. 6-8 in which we show our $\Omega$ values in the thresholds region for the $1-2\left(1 \mathrm{~s}{ }^{2} \mathrm{~S}_{1 / 2}-2 \mathrm{~s}{ }^{2} \mathrm{~S}_{1 / 2}\right), 1-3\left(1 \mathrm{~s}{ }^{2} \mathrm{~S}_{1 / 2}-2 \mathrm{p}{ }^{2} \mathrm{P}_{1 / 2}^{\circ}\right)$ and $2-3\left(2 \mathrm{~s}^{2} \mathrm{~S}_{1 / 2}-2 \mathrm{p}^{2} \mathrm{P}_{1 / 2}^{\circ}\right)$ transitions, respectively. It may be noted that in spite of the 2-3 transition being allowed, its resonance structure is significant as shown in Fig. 8.

Our calculated values of $\Upsilon$ are listed in Table 4 over a wide temperature range of $5.6 \leq \log T_{\mathrm{e}} \leq 7.4 \mathrm{~K}$, suitable for applications in astrophysical and other plasmas. As stated earlier in Sect. 1 there is no other similar calculation available in the literature with which to compare our results. The only $\Upsilon$ values available are on the CHIANTI database for transitions from the lowest two levels to higher excited levels. These values of $\Upsilon$ have been interpolated from our earlier $R$-matrix calculations in $L S$ coupling for $\mathrm{Ne} \mathrm{X}$

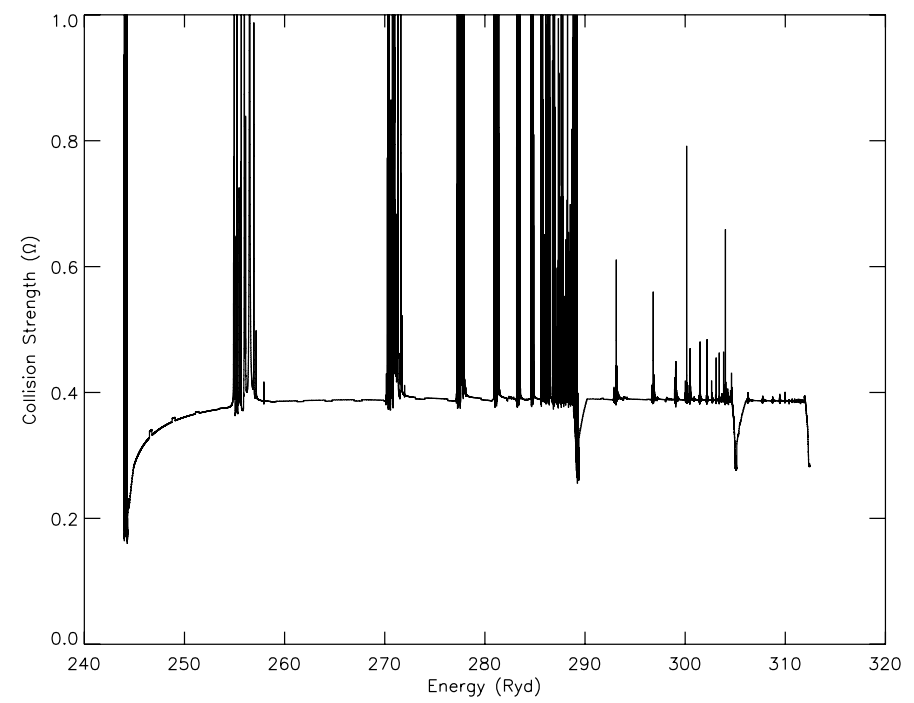

Fig. 8. Collision strengths for the $2 \mathrm{~s}^{2} \mathrm{~S}_{1 / 2}-2 \mathrm{p}{ }^{2} \mathrm{P}_{1 / 2}^{\circ}(2-3)$ transition of Ar XVIII.

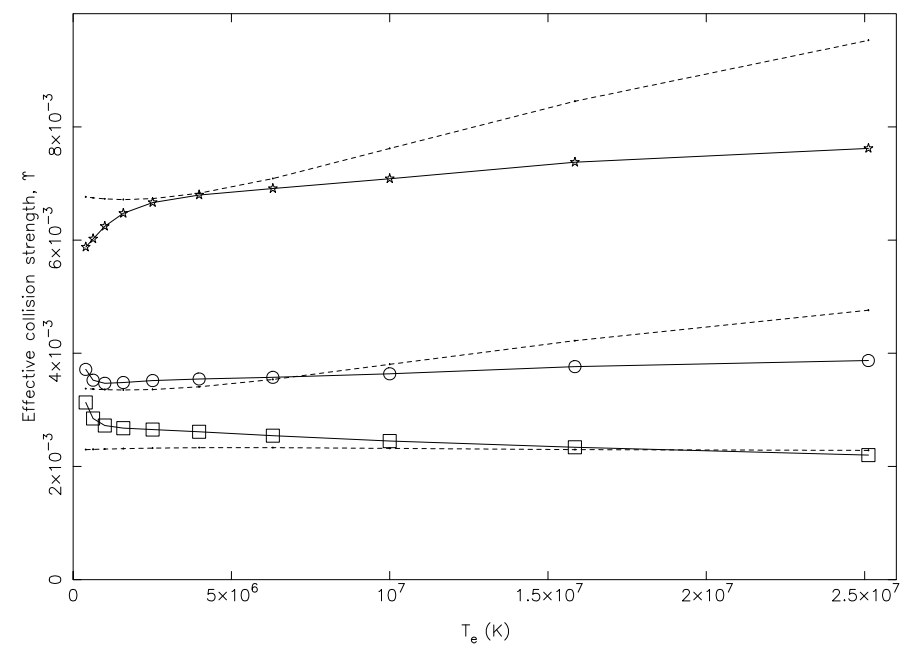

Fig. 9. Effective collision strengths for the 1-2 (squares: $1 \mathrm{~s}{ }^{2} \mathrm{~S}_{1 / 2}-2 \mathrm{~s}{ }^{2} \mathrm{~S}_{1 / 2}$ ), $1-3$ (circles: $1 \mathrm{~s}{ }^{2} \mathrm{~S}_{1 / 2}-2 \mathrm{p}{ }^{2} \mathrm{P}_{1 / 2}^{\circ}$ ) and $1-4$ (stars: $1 \mathrm{~s}{ }^{2} \mathrm{~S}_{1 / 2}-2 \mathrm{p}{ }^{2} \mathrm{P}_{3 / 2}^{\circ}$ ) transitions of Ar XVIII. Continuous curves are the present results from DARC and broken curves are from the CHIANTI database.

(Aggarwal \& Kingston 1991), Si XIV (Aggarwal \& Kingston 1992a), Ca XX (Aggarwal \& Kingston 1992b), and Fe XXVI (Aggarwal \& Kingston 1993).

A comparison of our values of $\Upsilon$ with those on the CHIANTI database shows differences of up to $40 \%$, except for two transitions, namely $1-23\left(1 \mathrm{~s}^{2} \mathrm{~S}_{1 / 2}-5 \mathrm{~g}{ }^{2} \mathrm{G}_{7 / 2}\right)$ and $1-25$ $\left(1 \mathrm{~s}{ }^{2} \mathrm{~S}_{1 / 2}-5 \mathrm{~g}{ }^{2} \mathrm{G}_{9 / 2}\right)$. For these two weak transitions $\left(\Upsilon \sim 10^{-7}\right)$ the differences are a factor of two over the entire temperature range. There are some transitions, such as 1-2, 1-10 and 1-11, for which the differences are noticeable towards the lower end of the temperature range. By contrast, for transitions, such as $1-3,2-5$ and $2-8$, the differences are primarily at higher temperatures. Some transitions, such as $1-12,15,16$, show discrepancies over the entire temperature range, but there are also some transitions, such as $1-5,1-13$ and $1-17$, for which the agreement is better than $10 \%$. To illustrate this, in Fig. 9 we compare the two sets of $\Upsilon$ for three transitions, namely $1-2\left(1 \mathrm{~s}{ }^{2} \mathrm{~S}_{1 / 2}-2 \mathrm{~s}{ }^{2} \mathrm{~S}_{1 / 2}\right), 1-3\left(1 \mathrm{~s}{ }^{2} \mathrm{~S}_{1 / 2}-2 \mathrm{p}{ }^{2} \mathrm{P}_{1 / 2}^{\circ}\right)$ and $1-4$ 
$\left(1 \mathrm{~s}^{2} \mathrm{~S}_{1 / 2}-2 \mathrm{p}^{2} \mathrm{P}_{3 / 2}^{\circ}\right)$. Considering that the CHIANTI data are based on the interpolation of earlier results in $L S$ coupling, differences with the present calculations are understandable. However, we recommend that the presently reported values of $\Upsilon$ should be adopted in all future data analysis.

\section{Conclusions}

In the present work, results for energy levels, radiative rates, collision strengths, and effective collision strengths for transitions among the lowest 25 levels of Ar XVIII have been presented for all transitions. Additionally, results for radiative rates have been presented for four types of transitions, namely E1, E2, M1 and M2. This complete dataset shall be very useful for modelling a variety of plasmas.

Additionally, our calculations have been performed in the $j j$ coupling scheme, CI (configuration interaction) and relativistic effects have been included while generating wavefunctions, and a wide range of partial waves has been adopted in order to achieve convergence in $\Omega$ values for a majority of transitions. Furthermore, resonances have been resolved in a fine energy mesh in order to improve the accuracy of the derived values of $\Upsilon$. Similarly, $\Omega$ have been computed over a wide energy range up to 800 Ryd in order to determine values of $\Upsilon$ up to a temperature of $10^{7.4} \mathrm{~K}$. Based on comparisons made among a variety of calculations, our energy levels are assessed to be accurate to $\sim 0.1 \%$, whereas the accuracy of other atomic parameters is probably better than $10 \%$.
Acknowledgements. This work has been financed by the Engineering and Physical Sciences Research Council (EPSRC) and the Science and Technology Facilities Council (STFC) of the UK. We would like to thank Dr. Patrick Norrington for providing his GRASP and DARC codes prior to publication, and FPK is grateful to AWE Aldermaston for the award of a William Penney Fellowship. Finally, KMA would like to thank the University of Miyazaki for the hospitality enjoyed during a visit in December 2007.

\section{References}

Aggarwal, K. M., \& Kingston, A. E. 1991, Phys. Scr., 44, 517 Aggarwal, K. M., \& Kingston, A. E. 1992a, Phys. Scr., 46, 193 Aggarwal, K. M., \& Kingston, A. E. 1992b, J. Phys. B, 25, 751 Aggarwal, K. M., \& Kingston, A. E. 1993, ApJS, 85, 187

Aggarwal, K. M., \& Keenan, F. P. 2005, A\&A, 441, 831

Aggarwal, K. M., Keenan, F. P., \& Nakazaki, S. 2005, A\&A, 436, 1141

Aggarwal, K. M., Hamada, K., Igarashi, A., et al. 2008a, A\&A, 484, 879

Aggarwal, K. M., Igarashi, A., Keenan, F. P., \& Nakazaki, S. 2008b, A\&A, 479, 585

Burgess, A., Hummer, D. G., \& Tully, J. A. 1970, Phil. Trans. Roy. Soc., 266 A, 225

Dere, K. P., Landi, E., Young, P. R., \& Del Zanna, G. 2001, ApJS, 134, 331

Grant, I. P., McKenzie, B. J., Norrington, P. H., Mayers, D. F., \& Pyper, N. C. 1980, Comput. Phys. Commun., 21, 207

Gu, M. F. 2003, ApJ, 582, 1241

Igarashi, A., Horiguchi, Y., Ohsaki, A., \& Nakazaki, S. 2003, J. Phys. Soc. Japan, 72,307

Igarashi, A., Ohsaki, A., \& Nakazaki, S. 2005, J. Phys. Soc. Japan, 74, 321

McKeown, K., Aggarwal, K. M., Keenan, F. P., \& Rose, S. J. 2004, Phys. Scr., 70,295

Parpia, F. A., \& Johnson, W. R. 1982, Phys. Rev. A, 26, 1142 